\title{
A Replication of "Motor and Visual Codes Interact to Facilitate Visuospatial Memory Performance (2007; Experiment 1)"
}

\author{
Vanessa P. Rowe a, Sébastien Lagacéa, Katherine Guérard ${ }^{\text {国, a }}$ \\ a École de psychologie, Université de Moncton
}

Abstract - The present study is a replication of Chum, Bekkering, Dodd, \& Pratt (2007). Motor and visual codes interact to facilitate visuospatial memory performance. Psychonomic Bulletin \& Review, 14, 1189-1193

Keywords " Visuospatial memory; Memory codes, Replication study.

Đ katherine.guerard@umoncton.ca

\section{Introduction}

Numerous researchers have investigated the influence of perception in spatial memory (Fischer \& Hoellen, 2004; Parmentier, Maybery, \& Jones, 2004). However, very few studies have examined whether actions play a role in the retention of spatial information. The objective of Chum, Bekkering, Dodd and Pratt (2007)'s study was to examine the effect of pointing on spatial memory and to determine if the activation of the motor system through actions can improve performance in a spatial memory task. Their results showed a recall advantage for items encoded through the perceptual and action systems compared to those encoded by means of the perceptual system only. Thus, their results support the idea that actions can have a facilitating effect on spatial working memory. Their results are in line with the embodied cognition framework (Barsalou, 1999), which suggests that cognition arises from the interaction between perception and action. This article inspired other researchers to further examine the interaction between perception and action in spatial memory (Dodd \& Shumbroski, 2009; Jones \& Martin, 2009; Rossi-Arnaud, Spataro, \& Longobardi, 2012).

In the present study, we replicated the first experiment of Chum et al. (2007). Participants had to memorize two spatial arrays presented successively on the computer screen. Each array comprised between three and five items presented successively in different spatial locations. For one array, participants were required to touch the items to be retained. The other array was encoded by means of visual processing only.

\section{Method}

\section{Participants}

Twenty-four undergraduate students from Université de Moncton participated in the experiment in exchange for a small honorarium. All reported normal or corrected-to-normal vision.

\section{Materials and Procedure}

The procedure was exactly the same as that used by Chum et al. (2007), except for the following details that were not specified in the method section. The stimuli were presented on a 18-inches Planar touch-screen using E-Prime 2.0. The items in each array were presented sequentially on the screen in random locations in a $5 \times 5$ invisible grid centered in the computer screen. Each cell of the grid sustained $2.3^{\circ} \times$ $2.3^{\circ}$ in visual angle. During the test phase, one of the two arrays reappeared and participants had to indicate whether the array was the same or different as the memorized array, by touching one of two green circles of $3.3^{\circ}$ of diameter located at the bottom right and left of the screen. The left circle contained the letter S for same and the right circle contained the letter D for different. The experimenter remained with participants during the experiment to ensure compliance with the instructions. 


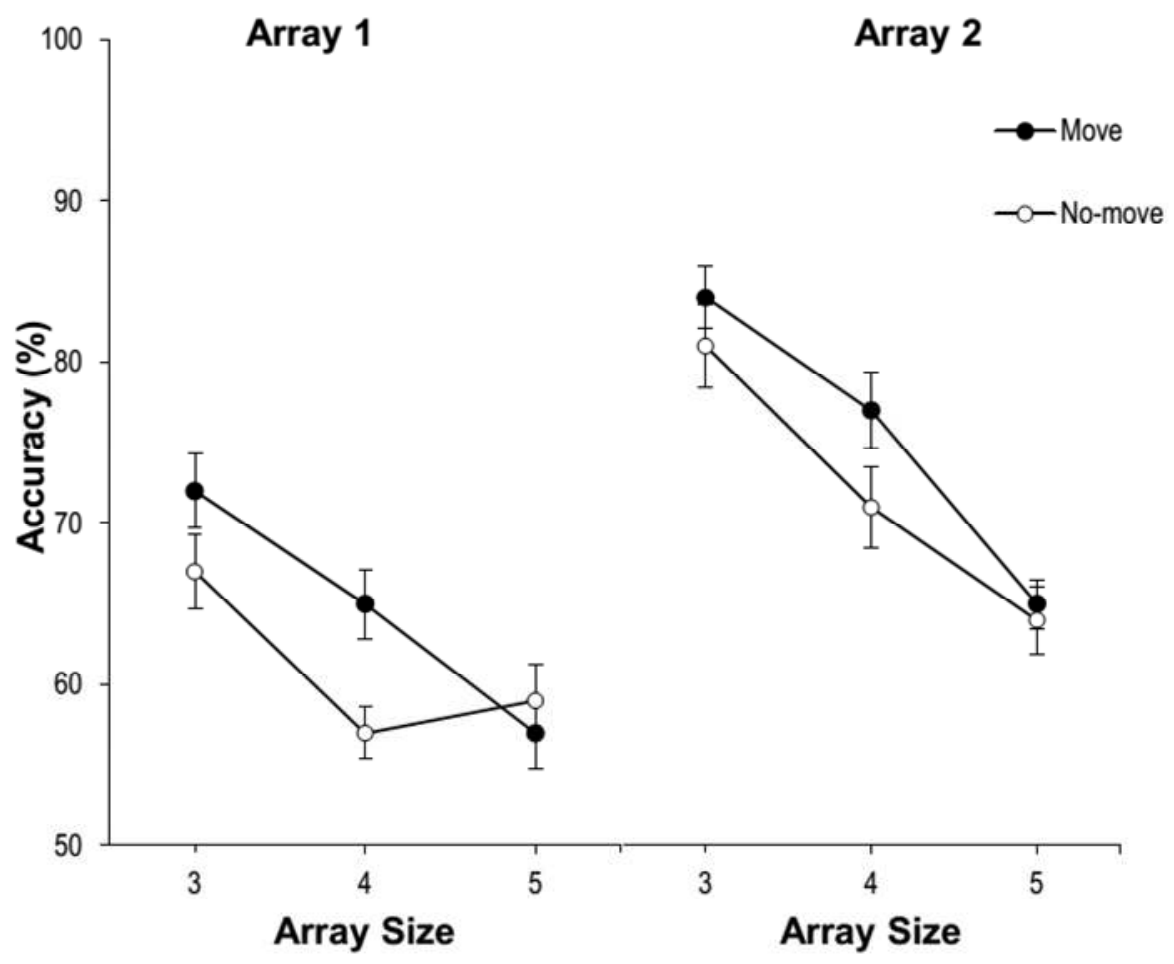

Figure 1 - Recognition accuracy as a function of array order, array size and pointing instructions

\section{Results}

Figure 1 presents recognition accuracy as a function of array order, array size and pointing instructions. A 2 (array order; first, second) $\times 2$ (pointing instructions; move, no move) $\times 3$ (array size; three, four, five) repeated-measures analysis of variance was performed on recognition accuracy. The results of this analysis are reported in Table 1 along with the results of Chum et al. (2007). ${ }^{1}$

\section{Discussion}

In the present replication, we obtained a pattern of results very similar to that reported by Chum et al. (2007). Qualitatively, the pattern of results presented in Figure 1 mirrors that presented in Chum et al.: pointing at the items during encoding seemed to improve spatial retention compared to a no-move condition. Although the main effect of pointing instructions did not reach significance in this replication ( $p=.06$ compared to $p=.01$ in Chum et al.'s Experiment 1), the effect sizes in both experiments are very similar, which suggests that pointing improved

1 These statistics were not available in the article, but were kindly provided by the corresponding author. spatial memory.

Moreover, the results point to a better recall for sequences presented in the second array compared to the first array and to a diminution of performance as a function of array size. At a statistical level, the significant main effects of array order and array size replicated their effects. Inspection of Figure 1 suggests that the interaction between pointing and array size is similar to that obtained by Chum et al., even though it did not reach significance in the present study. The effect sizes for that interaction in the two studies nevertheless suggest very similar patterns. Given the very similar results presented in Figure 1 and by Chum et al., the statistical differences are likely to be due to a higher variability in our sample of subjects. Considering the pattern exhibited in Figure 1 and the reported effect sizes, we believe our results replicated those of Chum et al. very closely.

\section{Authors' notes and acknowledgments}

This research was supported by discovery grants from the Natural Sciences and Engineering Research Council of Canada to Katherine Guérard. Correspondence concerning this article should be addressed to Katherine Guérard, École de psychologie, Université de Moncton, Moncton, New Brunswick, E1A 3E9, Canada. 
Table 1 - ANOVAs from Chum et al. and the present study

\begin{tabular}{lcccccccc}
\hline & \multicolumn{3}{c}{ Chum et al. (2007) } & & \multicolumn{3}{c}{ Replication } \\
\cline { 2 - 3 } \cline { 6 - 8 } Source & $F$ & $p$ & $\eta^{2}$ & & $F$ & $p$ & $\eta^{2} p$ \\
\hline Array order (AO) & 24.63 & .001 & .52 & & 31.60 & .001 & .58 \\
Pointing instructions (PI) & 7.6 & .01 & .25 & & 3.96 & .06 & .15 \\
Array size (AS) & 18.66 & .001 & .45 & & 25.66 & .001 & .53 \\
& & & & & & & \\
AO x PI & 0.002 & .96 & .00 & & 0.00 & .99 & .00 \\
AO x AS & 0.66 & .52 & .03 & & 1.08 & .35 & .05 \\
PI x AS & 3.64 & .03 & .14 & & 1.40 & .26 & .06 \\
& & & & & & & \\
AO x PI x AS & 0.002 & .99 & .00 & & 0.31 & .74 & .01 \\
\hline
\end{tabular}

E-mail: katherine.guerard@umoncton.ca

\section{References}

Barsalou, L. W. (1999). Perceptual symbol systems. Behavioral and Brain Sciences, 22, 577-660. doi:10.1017/S0140525X99002149

Chum, M., Bekkering, H., Dodd, M. D., \& Pratt, J. (2007). Motor and visual codes interact to facilitate visuospatial memory performance. Psychonomic Bulletin \& Review, 14, 1189-1193. doi:10.3758/ BF03193111

Dodd, M. D., \& Shumborski, S. (2009). Examining the influence of action on spatial working memory: The importance of selection. The Quarterly Journal of Experimental Psychology, 62, 1236-1247. doi:10.1080/17470210802439869
Fischer, M. H., \& Hoellen, N. (2004). Space- and objectbased attention depends on motor intention. Journal of General Psychology, 131, 365-377.

Jones, G. V., \& Martin, M. (2009). Spatial recall improved by retrieval enactment. Psychonomic Bulletin \& Review, 16, 524-528. doi:10.3758/PBR.16.3.524

Parmentier, F. B. R., Maybery, M. T., \& Jones, D. M. (2004). Temporal grouping in auditory spatial serial memory. Psychonomic Bulletin \& Review, 11, 501507. doi: $10.3758 / \mathrm{BF} 03196602$

Rossi-Arnaud, C., Spataro, P., \& Longobardi, E. (2012). Effects of pointing on the recall of simultaneous and sequential visuospatial arrays: A role for retrieval strategies? Psychological Research, 76, 699-712. doi:10.1007/s00426-011-0394-8

\section{Citation}

Rowe, V. P., Lagacé, S., \& Guérard, K. (2015). A Replication of "Motor and Visual Codes Interact to Facilitate Visuospatial Memory Performance (2007; Experiment 1)". The Quantitative Methods for Psychology, 11 (1), r1r3. 